\title{
Edema worsens target coverage in high-dose-rate interstitial brachytherapy of mobile tongue cancer: a report of two cases
}

\author{
Ken Yoshida, MD! Hideya Yamazaki, MD², Tadayuki Kotsuma, MD³. Hironori Akiyama, DDS4, Tadashi Takenaka, RTT², \\ Koji Masui, MD², Yasuo Yoshioka, MD5, Yasuo Uesugi, MD', Taiju Shimbo, MD', Nobuhiko Yoshikawa, MD!, \\ Hiroto Yoshioka, MD!, Takumi Arika, DDS?7, Eiichi Tanaka, MD³, Yoshifumi Narumi, MD' \\ 'Department of Radiology, Osaka Medical College, Osaka, ²Department of Radiology, Kyoto Prefectural University of Medicine, Kyoto, \\ ${ }^{3}$ Department of Radiation Oncology, National Hospital Organization Osaka National Hospital, Osaka, ${ }^{4}$ Department of Oral Radiology. \\ Osaka Dental University, Osaka, ${ }^{5}$ Department of Radiation Oncology, Osaka University Graduate School of Medicine, Osaka, ${ }^{6}$ Department \\ of Rehabilitation Sciences, Faculty of Allied Health Sciences, Kansai University of Welfare Sciences, Osaka, ${ }^{7}$ Department of Oral Surgery, \\ National Hospital Organization Osaka National Hospital, Osaka, Japan
}

\begin{abstract}
Purpose: We report our study on two patients to highlight the risk of underdosage of the clinical target volume (CTV) due to edema during high-dose-rate interstitial brachytherapy (HDR-ISBT) of mobile tongue cancer.

Material and methods: To treat the lateral side of the CTV, flexible applicator tubes were implanted on the mouth floor. Two-dimensional planning was performed using X-ray images for Case 1, and three-dimensional (3D) planning was performed using computed tomography (CT) for Case 2. Prescribed doses for both cases were 54 Gy in nine fractions.

Case reports: Case 1 was treated for cancer of the right lateral border of the tongue in 2005 . Tongue edema occurred after implantation, and part of the lateral border of the tongue protruded between the applicator tubes. Acute mucosal reaction abated in the protruded area earlier than in the other parts of the CTV. In this case, the tumor recurred in this area 5 months after the treatment. Case 2 was treated for cancer of the left lateral border of the tongue. Because tongue edema occurred in this case also, plastic splints were inserted between the applicator tubes to push the edematous region into the irradiated area. The mucosal surface of the CTV was covered by the $70 \%$ isodose, and $100 \%$ isodose line for before and after splint insertion. Local control of the tumor was achieved 4 years after treatment.

Discussion and conclusions: To ensure sufficient target coverage, 3D image-based planning using CT should be performed, followed by re-planning using repeated CT as needed. Also, the development of devices to prevent protrusion of the edematous tissue outside the target area will help to ensure the full dosing of CTV.
\end{abstract}

J Contemp Brachytherapy 2017; 9, 1: 66-70 DOI: https://doi.org/10.5114/jcb.2017.65163

Key words: edema, HDR, target coverage, tongue cancer.

\section{Purpose}

Radiotherapy is one of the standard treatment modalities for anterior mobile tongue cancer. Low-dose-rate (LDR) and high-dose-rate (HDR) interstitial brachytherapy (ISBT), with or without external beam radiotherapy (EBRT), shows a high rate of local control that is nearly equivalent to that of radical surgery $[1,2,3,4,5,6,7,8,9,10]$. We also showed that local control rates using HDR-ISBT for early mobile tongue cancer is an effective alternative treatment $[7,8]$.

In most institutes, a single treatment plan for dose optimization of mobile tongue brachytherapy is developed just after implantation. However, the dosimetry will change if there is any change in the geometry between the applicator positions and the clinical target volume (CTV) following treatment planning. In prostate brachytherapy, both edema and applicator displacement are considered as causes of underdosage or overdosage to the CTV and organs at risk (OAR) $[11,12,13,14]$. In case of prostate implants, the end of the applicator tip is inside the body and is not in a fixed position. Such that, the geometry between the applicator positions and CTV location will often change during treatment. However, applicator displacement in tongue brachytherapy is less of an issue because the applicator tip is fixed inside the oral cavity; thus, the geometry between the applicator and the CTV during
Address for correspondence: Ken Yoshida, MD, Department of Radiology, Osaka Medical College, 2-7, Daigakumachi, Takatsuki, Osaka 569-8686, Japan, phone: +81 72683 1221,

e-mail: rad113@osaka-med.ac.jp
Received: 14.09 .2016

Accepted: 14.12 .2016

Published: 28.02.2017 
treatment will not change. Tongue edema is a problem that is often observed during ISBT of the tongue because applicator implantation by itself can cause tongue edema $[15,16]$. Tongue edema can reduce target coverage because the treatment margin of brachytherapy is only 5-10 $\mathrm{mm}$ from gross tumor volume (GTV) $[10,17]$.

We report on how tongue edema in one case affected dosimetry to such an extent that local tumor recurrence occurred. We also describe a second case, in which tongue edema led to reduced tumor coverage, which was quantified using three-dimensional (3D) CT-based treatment planning.

\section{Case reports}

\section{Applicator implantation}

Implantation of applicators was performed under general anesthesia in both cases at National Hospital Organization Osaka National Hospital. To treat the lateral side of the CTV, a metallic open-ended needle (Bevel Needle ${ }^{\circledR}$, Elekta AB, Stockholm, Sweden) was inserted from the submandibular region and was implanted on the mouth floor or the lateral border of the tongue. Next, the needle was inserted into the vinyl template. After insertion of the needle, it was replaced by a flexible applicator tube (Flexible implant tube, $6 \mathrm{~F}$, single leader ${ }^{\circledR}$; Elekta $\mathrm{AB}$ ). To treat the medial side of the tongue, the open-ended needle was implanted to the dorsal surface of the tongue and inserted into the hole of the vinyl template. After that, a flexible applicator tube was replaced.

\section{Treatment planning and treatment}

For Case 1, we performed two-dimensional treatment planning using two orthogonal X-ray images to confirm applicator positions. Prescribed doses were delivered to $5 \mathrm{~mm}$ from the applicator. Titanium markers were implanted at the edge of the tumor to help draw the tumor contour and position on treatment planning software (PLATO, Elekta AB). For Case 2, treatment planning was conducted using 3D CT. We drew the contour of the tongue and mandible in CT. The contour of the CTV was identified using not only CT but also by employing titanium markers and perioperative examinations (inspection, palpation, and intraoral ultrasonography). The extent of the tumor on the tongue's surface was also evaluated by staining with Lugol's iodine during implantation and by placement of metal markers.

We defined the CTV as an equivalent to GTV. We used a modified Paris system with computer optimization (geometrical optimization) and delivered prescribed doses to $85 \%$ of the basal dose at first. Subsequently, we always modified the isodose shape manually and sometimes changed the \% basal dose to adequately cover the CTV by isodose lines of the prescribed dose (PD) without excessive doses to OARs [18]. Prescribed dose was 54 Gy per nine fractions in 7 days. Biological effective dose $(\alpha / \beta=10)$ was $86.4 \mathrm{~Gy}$, and equivalent total dose in 2 Gy fractions $\left(\mathrm{EQD}_{2}\right)$ was $72 \mathrm{~Gy}$. Our planning aim was that the CTV was covered by $100 \%$ of PD. We used microSelectron-HDR ${ }^{\circledR}$ (Elekta $\mathrm{AB}$ ) with an ${ }^{192}$ Ir source.
Source strength was 249.2 GBq and 282.6 GBq for Case 1 and Case 2, respectively.

\section{Case 1}

A 55-year-old female patient had a squamous cell carcinoma on the right lateral border of her tongue that was staged cT2N0M0 using the 2002 UICC classification (Figure 1A). She underwent HDR-ISBT as monotherapy. Two plane implantation with 8 flexible applicator tubes was performed in November 2005. Tongue edema developed the day after implantation, and a part of the lateral border of the tongue protruded between two of the applicator tubes (Figure 1B). She had an acute mucosal reaction to the therapy, with exudative mucositis peaking 13 days after treatment; however, a part of the protruding area had no white coating (Figure 1C). After 23 days of treatment, the coating disappeared from nearly all the protruding area (Figure 1D). Five months after the treatment, the tumor recurred in this area (Figure 1E). Biopsy was performed and histological finding showed squamous cell carcinoma. She received salvage surgery and tumor was controlled until she died by intercurrent disease 122 months after salvage surgery.

\section{Case 2}

A 47-year-old male patient had a squamous cell carcinoma on the left lateral border that was staged cT2N0M0 using the 2002 UICC classification (Figure 2A). Two plane implantation with 9 flexible applicator tubes was performed in October 2010. We performed 3D CT-planning and delivered 6 Gy as $\mathrm{D}_{100}(\mathrm{CTV}=\mathrm{GTV})$. $\mathrm{D}_{90}(\mathrm{CTV})$ was $6.9 \mathrm{~Gy}$. If $5 \mathrm{~mm}$ margin will add to the CTV, $\mathrm{D}_{100}$ (CTV) and $\mathrm{D}_{90}(\mathrm{CTV})$ were $4.8 \mathrm{~Gy}$ and $6.5 \mathrm{~Gy}$, respectively. If $10 \mathrm{~mm}$ margin will add to the CTV, $\mathrm{D}_{100}(\mathrm{CTV})$ and $\mathrm{D}_{90}$ (CTV) were 3.5 Gy and $5.3 \mathrm{~Gy}$, respectively. This patient also developed tongue edema the day after implantation (Figure 2B); therefore, two plastic splints were inserted between applicator tubes to push the edematous tongue into the irradiated area (Figure 2C).

Figures 2D and 2E show the isodose distribution curves before and after splint insertion. The mucosal surface of the CTV was covered by the $70 \%$ isodose line before splint insertion, but it was covered by the $100 \%$ isodose line after splint insertion. Local control of tumor recurrence was achieved until the patient's death by neck metastasis 4 years after the treatment.

\section{Discussion}

Because ISBT can deliver higher doses of radiation to more localized regions than EBRT, better rates of local control in cases of mobile tongue carcinoma were expected, and the rapid dose fall-off from applicators could maintain complication rates within tolerable levels. However, if unexpected tongue edema occurs, dose fall-off will become a cause of underdosage to the CTV. Schultze et al. [15] investigated tongue edema in 51 patients with head and neck cancers (most patients had a base of tongue cancer and mobile tongue cancer was not included), and found that applicator-induced edem- 

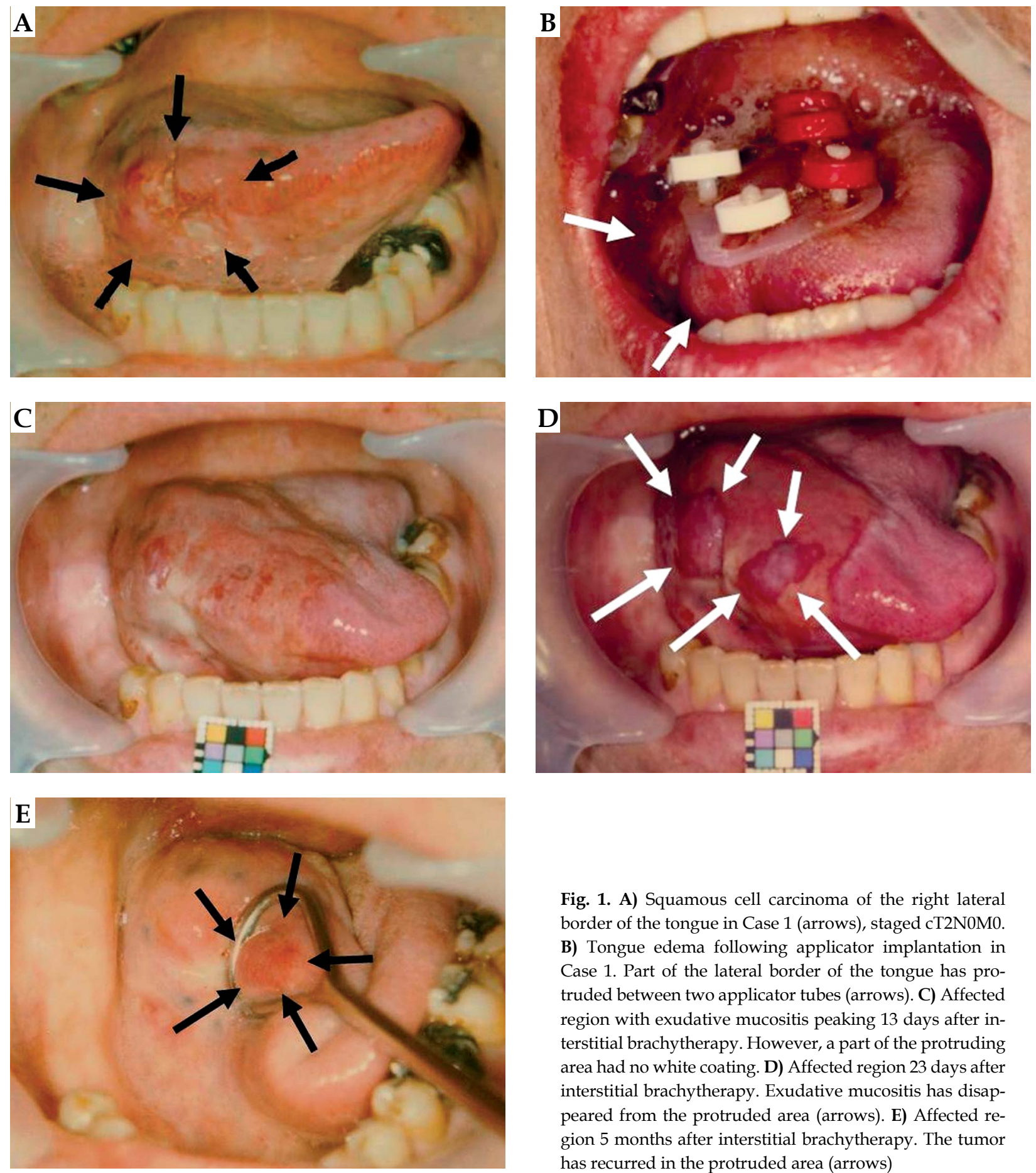

atous deviation of tongue width was $6 \mathrm{~mm}$, measured using magnetic resonance imaging (MRI). American Brachytherapy Society (ABS) recommendations showed that a smaller dose per fraction may be reduced normal tissue injury. Our single fraction doses were $6 \mathrm{~Gy}$ and $\mathrm{D}_{100}(\mathrm{CTV})$ was $6 \mathrm{~Gy}$. However, $\mathrm{D}_{90}$ (CTV) was 6.9 Gy. Furthermore, $\mathrm{D}_{90}(\mathrm{CTV})$ and $\mathrm{D}_{100}(\mathrm{CTV})$ change 3.5-6.5 Gy if CTV margin of $5-10 \mathrm{~mm}$ was added. In future, we should investigate which DVH value showed the highest correlation with tongue edema. Our total prescribed doses were $54 \mathrm{~Gy}$ and $\mathrm{EQD}_{2}(\alpha / \beta=10)$ was $72 \mathrm{~Gy}$. It is

Fig. 1. A) Squamous cell carcinoma of the right lateral border of the tongue in Case 1 (arrows), staged cT2N0M0. B) Tongue edema following applicator implantation in Case 1. Part of the lateral border of the tongue has protruded between two applicator tubes (arrows). C) Affected region with exudative mucositis peaking 13 days after interstitial brachytherapy. However, a part of the protruding area had no white coating. D) Affected region 23 days after interstitial brachytherapy. Exudative mucositis has disappeared from the protruded area (arrows). E) Affected region 5 months after interstitial brachytherapy. The tumor has recurred in the protruded area (arrows)

relatively higher than 54-80 Gy for the other series [16]. However, it is also a same problem showing above. Implant volume may also be a risk factor of tongue edema. If implant volume becomes larger, both traumatic and irradiation damages become larger. We implanted two plane implantations for these 2 cases, and it led to not much implant volume because our standard technique was two or more plane implantations [19]. Further investigation will also be necessary.

American Brachytherapy Society recommendations also showed that corticosteroids may be used to reduce 

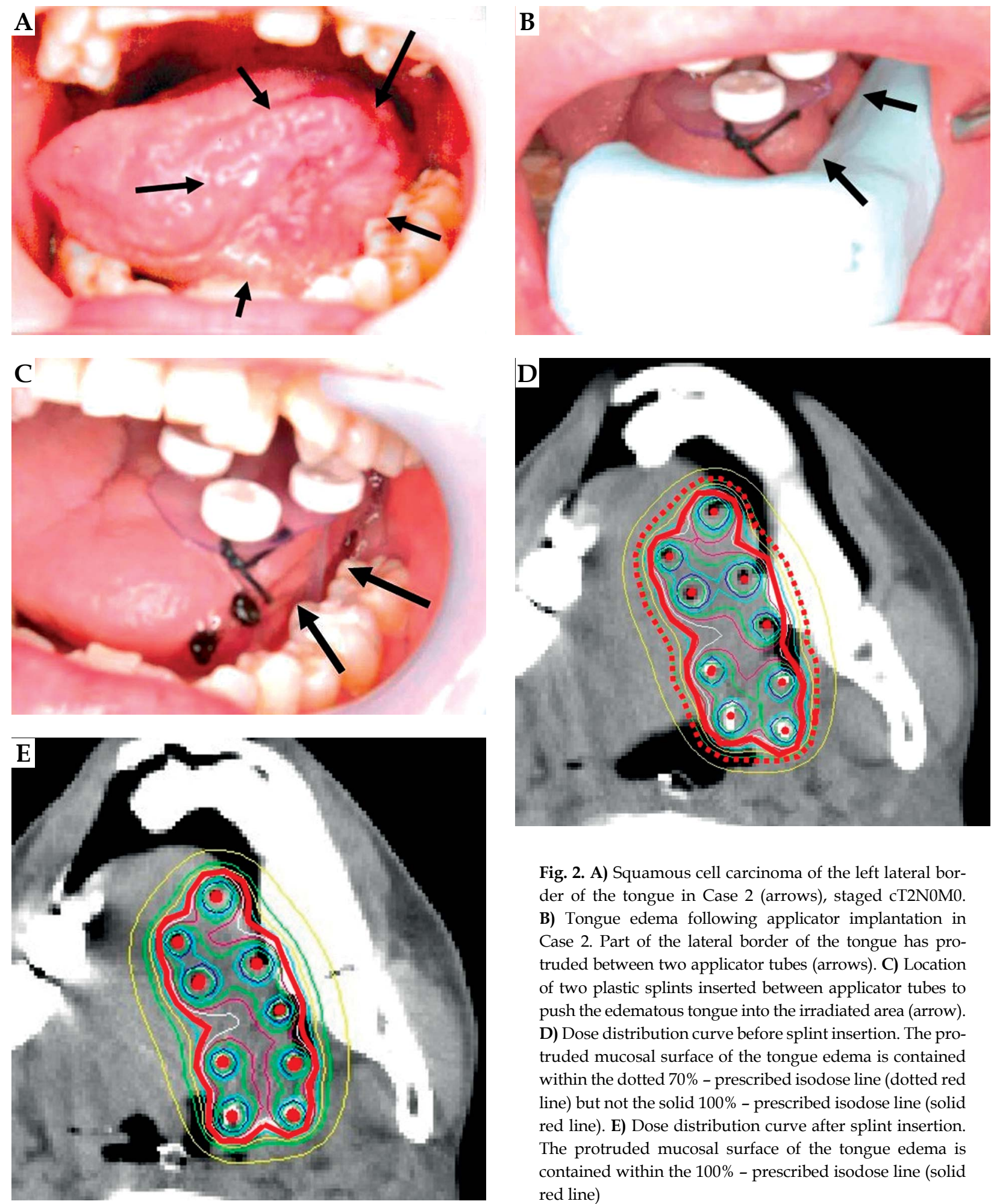

Fig. 2. A) Squamous cell carcinoma of the left lateral border of the tongue in Case 2 (arrows), staged cT2N0M0. B) Tongue edema following applicator implantation in Case 2. Part of the lateral border of the tongue has protruded between two applicator tubes (arrows). C) Location of two plastic splints inserted between applicator tubes to push the edematous tongue into the irradiated area (arrow). D) Dose distribution curve before splint insertion. The protruded mucosal surface of the tongue edema is contained within the dotted $70 \%$ - prescribed isodose line (dotted red line) but not the solid 100\% - prescribed isodose line (solid red line). E) Dose distribution curve after splint insertion. The protruded mucosal surface of the tongue edema is contained within the $100 \%$ - prescribed isodose line (solid red line)

postoperative swelling. We infused dexamethasone sodium phostate $(8 \mathrm{mg})$ intravenously to prevent tongue edema during implantation. However, severe tongue edema sometimes occurred. And so, we must consider the way to solve this problem.

To prevent underdosing related to edema, 3D imagebased planning using $\mathrm{CT}$ should be performed to evalu-

ate the dose distribution of the CTV and OARs [20,21]. Because edematous changes of the tongue may occur after first CT planning, CT should be repeated every day during the treatment period. And, re-planning should be performed to keep adequate dose coverage for the CTV. However, because treatment re-planning is time consuming, it would be desirable to have a device that could 
counter edema-related protrusion of tissues outside the target area. We contrived a means, by which edematous tissue could be contained between the applicator tubes by using a splint insertion for Case 2. We are in the process of developing a specific device for this purpose and will report on it in the near future.

\section{Conclusions}

To ensure sufficient target coverage, 3D image-based planning using CT should be performed, followed by re-planning using repeated $\mathrm{CT}$ as needed. Also, the development of devices to prevent protrusion of the edematous tissue outside the target area will help ensure full dosing of the CTV.

\section{Acknowledgments}

This work was supported by JSPS Grant-in-Aid for Scientific Research (KAKENHI) (C) Grant Number 25461931, Ministry of Health, Labour and Welfare Grant-in-Aid for Scientific Research (KAKENHI) Grant Number 26270801 and Japan mHDR Research Fund.

We would like to thank Mineo Yoshida, MD, Katsuhito Kubo, RTT, the staff of the departments of Radiation oncology for helping us in many ways during the completion of this paper.

\section{Disclosure}

Authors report no conflict of interest.

\section{References}

1. Mazeron JJ, Crook JM, Benck V et al. Iridium 192 implantation of $\mathrm{T} 1$ and $\mathrm{T} 2$ carcinomas of the mobile tongue. Int J Radiat Oncol Biol Phys 1990; 19: 1369-1376.

2. Pernot M, Malissard L, Aletti P et al. Iridium-192 brachytherapy in the management of $147 \mathrm{~T} 2 \mathrm{~N} 0$ oral tongue carcinomas treated with irradiation alone: comparison of two treatment techniques. Radiother Oncol 1992; 23: 223-228.

3. Shibuya H, Hoshina M, Takeda $M$ et al. Brachytherapy for stage I \& II oral tongue cancer: an analysis of past cases focusing on control and complications. Int J Radiat Oncol Biol Phys 1993; 26: 51-58.

4. Hareyama M, Nishio M, Saito A et al. Results of cesium needle interstitial implantation for carcinoma of the oral tongue. Int J Radiat Oncol Biol Phys 1993; 25: 29-34.

5. Fujita M, Hirokawa $Y$, Kashiwado $K$ et al. Interstitial brachytherapy for stage I and II squamous cell carcinoma of the oral tongue: factors influencing local control and soft tissue complication. Int J Radiat Oncol Biol Phys 1999; 44: 767-775.

6. Shigematsu $\mathrm{Y}$, Masaki N, Ikeda $\mathrm{H}$ et al. Current status and future of brachytherapy. Gan No Rinsho 1983; 29: 695-701 [Article in Japanese].

7. Inoue $\mathrm{T}$, Teshima $\mathrm{T}$, Murayama $\mathrm{S}$ et al. Phase III trial of high and low dose rate interstitial radiotherapy for early oral tongue cancer. Int J Radiat Oncol Biol Phys 1996; 36: 12011204.

8. Inoue T, Yoshida K, Yoshioka Y et al. Phase III trial of high- vs. low-dose-rate interstitial radiotherapy for early oral tongue cancer. Int J Radiat Oncol Biol Phys 2001; 51: 171-175.

9. Kovács G. Modern head and neck brachytherapy: from radium towards intensity modulated interventional brachytherapy. J Contemp Brachytherapy 2015; 6: 404-416.
10. Bansal A, Ghoshal S, Oinam AS et al. High-dose-rate interstitial brachytherapy in early stage oral tongue cancer - 15-year experience from a tertiary care institute. J Contemp Brachytherapy 2016; 8: 56-65.

11. Martinez AA, Pataki I, Edmundson G et al. Phase II prospective study of the use of conformal high-dose-rate brachytherapy as monotherapy for the treatment of favorable stage prostate cancer: a feasibility report. Int J Radiat Oncol Biol Phys 2001; 49: 61-69.

12. Damore SJ, Syed AM, Puthawala AA et al. Needle displacement during HDR brachytherapy in the treatment of prostate cancer. Int J Radiat Oncol Biol Phys 2000; 46: 1205-1211.

13. Cury FL, Duclos M, Aprikian A et al. Prostate gland edema after single-fraction high-dose rate brachytherapy before external beam radiation therapy. Brachytherapy 2010; 9: 208-212.

14. Kiffer JD, Schumer WA, Mantle CA et al. Impact of oedema on implant geometry and dosimetry for temporary high dose rate brachytherapy of the prostate. Australas Radiol 2003; 47: 172-176.

15. Schultze J, Schneider R, Kimmig B et al. Interstitial brachytherapy in the head and neck region: when has imaging for treatment planning to be performed? Rontgenpraxis 2002; 54 : 232-237.

16. Nag S, Cano ER, Demanes DJ et al. The American Brachytherapy Society recommendation for high-dose-rate brachytherapy for head-and-neck carcinoma. Int J Radiat Oncol Biol Phys 2001; 50: 1190-1198.

17. Mazeron JJ, Ardiet JM, Haie-Meder C et al. GEC-ESTRO recommendations for brachytherapy for head and neck squamous cell carcinomas. Radiother Oncol 2009; 91: 150-156.

18. Yoshida K, Nose T, Koizumi M et al. The usefulness of metal markers for CTV-based dose prescription in high-dose-rate interstitial brachytherapy. J Jpn Soc Ther Radiol Oncol 2002; 13: $253-260$

19. Akiyama H, Yoshida K, Yamazaki H et al. High-dose-rate interstitial brachytherapy for mobile tongue cancer: preliminary results of a dose reduction trial. J Contemp Brachytherapy 2014; 6: 10-14.

20. Major T, Polgár C, Mangel L et al. CT based conformal brachytherapy treatment planning. Magy Onkol 2000; 44: 109-115 [Article in Hungarian].

21. Yoshida K, Takenaka T, Akiyama $\mathrm{H}$ et al. Three-dimensional image-based high-dose-rate interstitial brachytherapy for mobile tongue cancer. J Radiat Res 2014; 55: 154-161. 